\title{
Pharmakotherapie bei Schwerem Akutem Respiratorischem Syndrom (SARS)
}

\author{
Pharmacotherapy of Severe Acute Respiratory Syndrome (SARS)
}

Autoren

Institute
L. von Hagen' ${ }^{1}$, M. W. Pletz ${ }^{1}$, N. Dickgreber ${ }^{1}$, H. Golpon ${ }^{1}$, T. T. Bauer ${ }^{2}$, P. Zabel ${ }^{3}$, T. Welte ${ }^{1}$, D. A. Groneberg ${ }^{1}$

Die Institutsangaben sind am Ende des Beitrags gelistet. eingereicht 10.7. 2006

akzeptiert 26. 7. 2006

Bibliografie

DOI $10.1055 / \mathrm{s}-2006-944278$

Pneumologie 2006; 60; 694-700

(c) Georg Thieme Verlag KG

Stuttgart $\cdot$ New York

ISSN 0934-8387

\section{Korrespondenzadresse \\ Univ.-Prof. Dr. med. David Groneberg}

Zentrum Innere Medizin, Abteilung Pneumologie, Medizinische Hochschule Hannover

Carl-Neuberg-Str. 1 OE6870 30625 Hannover

groneberg.david@mh-hannover.de

Serienherausgeber

T. Schaberg

S. Ewig

\section{Zusammenfassung}

Das schwere akute respiratorische Syndrom (SARS) ist die erste bedeutende neue Infektionskrankheit dieses Jahrtausends und wird durch das SARS-Coronavirus (SARS-CoV) hervorgerufen. SARS geht mit einer erheblichen Morbidität und Mortalität einher und trat erstmals als Epidemie von 2002-2003 auf. Bis heute ist noch kein spezifisch wirksames Therapeutikum gegen das SARS-Coronavirus gefunden worden. Aufgrund des schnellen Voranschreitens der Epidemie 2002-2003 war es nicht möglich, multizentrierte, kontrollierte, randomisierte Studien durchzuführen. Daher existieren noch keine allgemeingültigen therapeutischen Richtlinien für diese neue Viruserkrankung. Seit dem Ausbruch von SARS untersuchen Wissenschaftler potenziell wirksame Substanzen gegen das Virus hauptsächlich mittels In-vitro-Tests und an Tiermodellen. Die vorliegende Arbeit analysiert die derzeit zur Pharmakotherapie vorhandenen Invitro- und In-vivo-Ergebnisse.

\section{Einleitung}

Das schwere akute respiratorische Syndrom (SARS) ist die erste neue Infektionskrankheit dieses Jahrtausends. Es geht mit einer erheblichen Morbidität und Mortalität einher und trat erstmals als Epidemie 2002/2003 in Südchina auf [1-5]. Aufgrund des internationalen Reiseverkehrs breitete sich die durch das SARS-Coronavirus ausgelöste Erkrankung global aus und führte zu insgesamt 8096 möglichen Fälle von SARS und 774 Toten in 29 Ländern [6-15]. Dabei traten über $90 \%$ der Fälle in China und Hongkong auf $[3,4]$, und die Erkrankung endete oft in einem ARDS [16].

\section{Abstract}

Severe acute respiratory syndrome (SARS) constitutes the first new infectious disease of the current millennium. It is caused by the novel SARSCoronavirus (SARS-CoV). SARS is related to a high morbidity and mortality and first appeared during an epidemic in 2002-2003. To date no specific therapy against the SARS-CoV is available. Due to the rapid spread of SARS during the epidemics in 2002-2003, randomised and controlled multicentre studies were not performed. Therefore, general guidelines have not been developed. Since the outbreak, scientists have been testing potential antiviral substances using in vitro and animal models. This study analyses the presently available in vitro and in vivo data on the pharmacotherapy of SARS.
Bis heute ist noch keine spezifisch wirksame Therapie gegen das SARS-Coronavirus gefunden, welches ein RNA-Virus darstellt und die Kochschen Postulate erfüllt [17-22]. Aufgrund des schnellen Voranschreitens der Epidemie 20022003 [23,24] war es nicht möglich, multizentrierte, kontrollierte, randomisierte Studien durchzuführen. Daher existieren noch keine allgemeingültigen therapeutischen Richtlinien für diese neue Viruserkrankung. Seit dem Ausbruch von SARS untersuchen Wissenschaftler potenziell wirksame Substanzen gegen das Virus hauptsächlich mittels in vitro Tests und an Tiermodellen. Die vorliegende Arbeit analysiert die derzeit zur Thematik erschienenen Studien. 


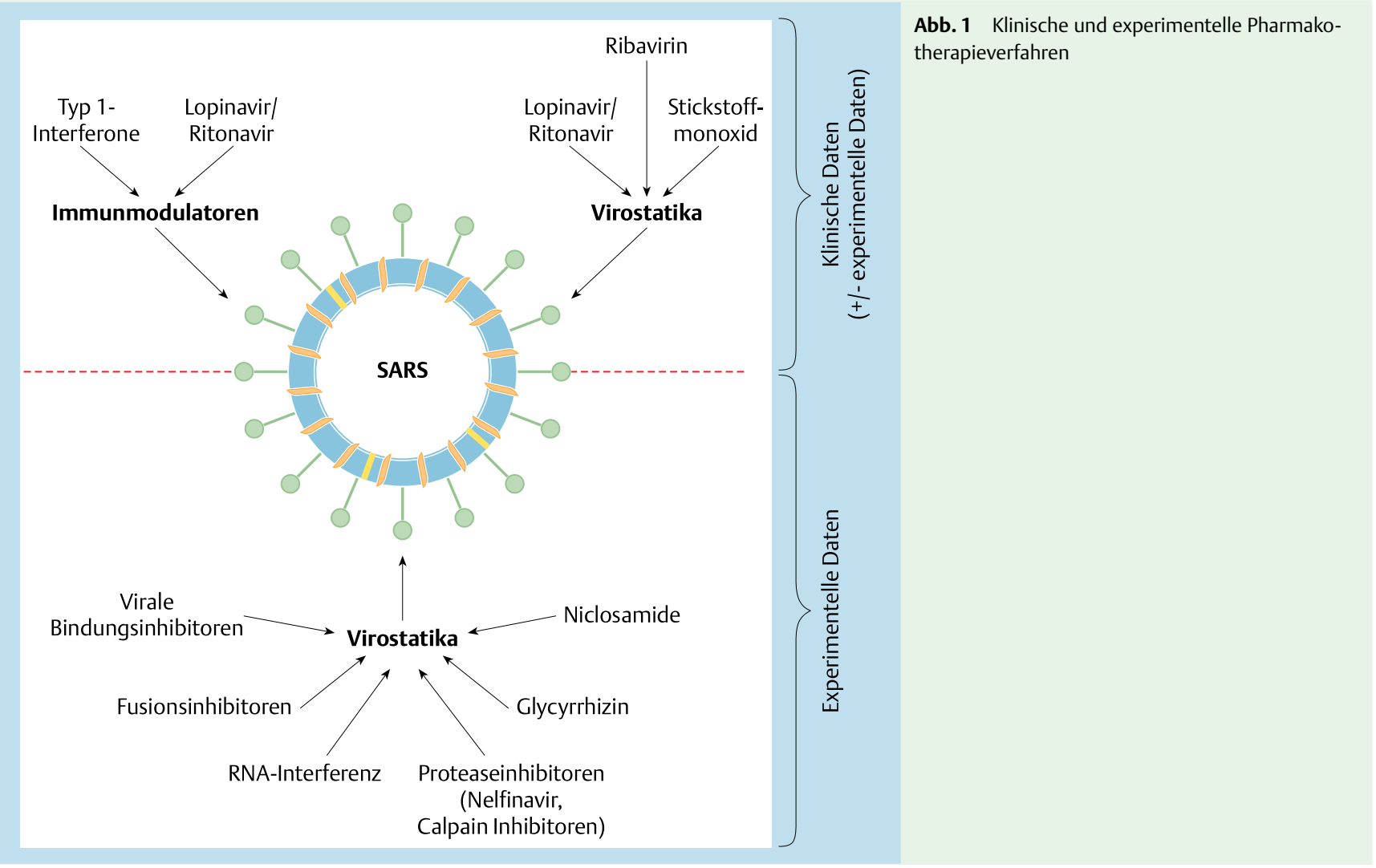

\section{Material und Methode}

Das Ziel der Arbeit ist es, mithilfe von Literaturrecherche den aktuellen Forschungsstand zu effizienten Pharmakotherapiemöglichkeiten von SARS zusammenzufassen. Die Arbeit soll einen Überblick über die In-vitro- und In-vivo-Daten geben ( Tab. 1), die die Wirksamkeit bisher eingesetzter oder vorgeschlagener Substanzen gegen SARS evaluieren. Auch die klinischen Erfahrungen, die während der SARS-Epidemie mit verschiedenen Therapeutika gemacht wurden, werden zusammenfassend dargestellt. Außerdem zeigt die Arbeit die Fortschritte, die bis heute in der Entwicklung von Impfstoffen gemacht wurden.

Es wurde eine Literatursuche in der medizinischen Datenbank Medline mittels Pubmed durchgeführt (2006-02-25). Als Suchwörter wurden zunächst „SARS AND treatment“ in der Freitextsuche eingegeben. Es erfolgte dann eine weitere Analyse aller Abstracts.

\section{Ergebnisse}

Die PubMed-Analyse ergab eine Trefferzahl von 1802 Einträgen. Nach der Durchsicht der Abstracts der einzelnen Artikel (Übertragung von Medline in Endnote) wurden die relevanten Arbeiten im Volltext analysiert. Die Pharmakotherapieansätze lassen sich untergliedern in Antibiotika, Virostatika oder Immunmodulatoren ( Abb. 1). Die meisten Therapiestrategien sind nicht evidenzbasiert und teilweise basierend auf präklinischen bzw. In-vitro-Daten ( Tab. 1).

\section{Antibiotika}

Während des Ausbruchs von SARS 2002/2003 wurden potenzielle SARS-Patienten initial mit Breitspektrumantibiotika be- handelt, die gegen die typischen bakteriellen Ursachen ambulant erworbener Pneumonien wirksam sind. Die Anwendung eines Breitspektrumantibiotikums plus einem Makrolid wird heute noch bei ersten Anzeichen einer SARS-Erkrankung empfohlen, da die initialen Symptome unspezifisch sind. Seitdem das SARSCoronavirus identifiziert wurde und es keine Anzeichen dafür gibt, dass Antibiotika zu einer klinischen Verbesserung führen, ist eine Behandlung mit Antibiotika jedoch nicht mehr indiziert.

\section{Virostatika - Ribavirin}

Ribavirin wurde bereits vor der Identifikation des SARS verursachenden Erregers empirisch als anti-SARS-Therapeutikum eingesetzt [25]. Bis heute existiert jedoch noch keine Studie, die die Wirksamkeit von Ribavirin in der Therapie von SARS eindeutig nachweist.

Ribavirin ist ein synthetisches Nukleosidanalogon. Es blockiert u. a. das Enzym Guanylyltransferase und hemmt so die Ausbildung der 5-cap-Struktur an viralen und eukaryoten mRNAs. Ribavirin wirkt daher nicht virusspezifisch, so dass es auch zu massiven Schädigungen von Wirtszellen kommen kann. Ribavirin ist gegen ein breites Spektrum von RNA- und DNA-Viren wirksam $[26,27]$.

Die bisher durchgeführten Fallstudien untersuchten die Wirkung von Ribavirin nur in Kombination mit weiteren Therapeutika. So zeigten Studien radiologische und symptomatische Verbesserungen bei Patienten, die mit einer Kombinationstherapie aus Ribavirin und Steroiden behandelt wurden [28,29]. Ohne den Einbezug einer Vergleichsgruppe ist es jedoch schwierig festzustellen, ob die Verbesserungen aufgrund der Therapie mit Ribavirin, mit Steroiden, der Kombination aus beiden Präparationen oder dem natürlichen Verlauf der Erkrankung eintraten. Eine Studie zeigte, dass ein verzögerter Therapiebeginn mit einer Kombination aus Ribavirin und Steroiden einen Risikofaktor für 


\begin{tabular}{|c|c|c|}
\hline Gruppe & Substanzen & Beispiele \\
\hline Antibiotika & Breitspektrumantibiotika & \\
\hline \multirow[t]{5}{*}{ Virostatika } & Ribavirin & \\
\hline & Proteaseinhibitoren & Lopinavir, Ritonavir, Nelfinavir \\
\hline & Bindungsinhibitoren & Anti-S1 Antikörper \\
\hline & Fusionsinhibitoren & Peptid CP1 \\
\hline & RNA-Interferenz & Spike Gen iRNA \\
\hline \multirow[t]{2}{*}{ Immunmodulatoren } & Steroide & \\
\hline & Interferone & Interferon alfacon 1 \\
\hline TCM & Glycyrrhizin & \\
\hline \multirow[t]{2}{*}{ Andere } & Stickstoffmonoxid & \\
\hline & Niclosamid & \\
\hline
\end{tabular}

Tab. 1 Anti-SARS-Therapiestrategien (nicht evidenzbasiert, teilweise präklinisch/in vitro). Abk. TCM Traditionelle Chinesische Medizin die Entwicklung eines schweren Verlaufes der Erkrankung darstellte. Auch diese Studie unterschied jedoch nicht zwischen einer verzögerten Gabe von Ribavirin und der verzögerten Gabe von Steroiden [30]. Es kann daher keine eindeutige Aussage über die Wirksamkeit von Ribavirin als Einzeltherapeutikum gemacht werden.

Eine randomisierte klinische Studie aus Guangdong kam zu dem Ergebnis, dass die Effektivität einer Therapie mit niedrig dosiertem Ribavirin (400-600 mg/dl) deutlich geringer war, als die Effektivität einer frühen aggressiven Therapie mit Steroiden und Interferon alfa [31]. Allerdings fehlte auch bei dieser Studie eine Kontrollgruppe, so dass keine eindeutigen Schlüsse zu der Wirkung einer Ribavirin-Therapie im Vergleich mit einem Therapieverzicht gezogen werden können.

In vitro-Tests zeigten, dass Ribavirin nicht in der Lage war, die Replikation des SARS-CoV in für eine erfolgreiche Therapie benötigter Konzentration zu hemmen [32,33].

Bei verstorbenen SARS-Patienten, die zuvor mit Ribavirin behandelt wurden, stellte man in Nachuntersuchungen eine hohe Viruslast fest [34].

Die Ergebnisse zeigen insgesamt, dass Ribavirin, wenn überhaupt, nur eine geringe therapeutische Wirkung gegen das SARS-CoV aufzeigt.

Dies ist insbesondere von Bedeutung, wenn man die nicht unerheblichen Nebenwirkungen betrachtet, die durch Ribavirin verursacht wurden. Knowles u. Mitarb. berichteten über ein Kollektiv von 110 möglichen SARS-Patienten, die mit Ribavirin behandelt wurden [35].

$61 \%$ dieser Patienten entwickelten Symptome einer hämolytischen Anämie; bei 58\% der Erkrankten wurde eine Hypokalziämie, bei $46 \%$ eine Hypomagnesiämie festgestellt.

\section{Virostatika - Proteaseinhibitoren}

Die Kombinationstherapie aus den Proteaseinhibitoren Lopinavir und Ritonavir stellte zu Anfang eine vielversprechende Therapiemöglichkeit dar, nachdem in vitro Studien die antivirale Aktivität dieser Substanzen gegen das SARS-Coronavirus nachgewiesen hatten [36,37].

Während der Zeit des Ausbruchs von SARS wurde diese Kombinationstherapie seltener eingesetzt als Ribavirin.

Chan u. Mitarb. [37] verglichen die Ergebnisse einer Kombinationstherapie mit Lopinavir/Ritonavir - als initiale Therapie und als Notfalltherapeutikum - mit Kontrollgruppen.

Alle Patienten wurden nach einem standardisierten Schema mit Steroiden und Ribavirin behandelt. Die zusätzliche initiale Therapie mit Lopinavir/Ritonavir zeigte im Vergleich zu den Kontrollgruppen eine statistisch signifikante Reduktion der Mortalitätsrate und der Intubationsrate $(p<0,05)$. Hingegen zeigte die
Patientengruppe mit Lopinavir/Ritonavir als Notfalltherapeutikum keinen statistisch signifikanten Unterschied in diesen Endpunkten. Auch Chu u. Mitarb. [36] verglichen die Therapie mit Lopinavir/Ritonavir mit Kontrollgruppen; allen Patienten wurden, ähnlich dem Schema der Chan-Studie, Ribavirin und Steroide verabreicht. Schwere Krankheitsverläufe (Entwicklung eines akuten Lungenversagens [ARDS] oder Tod innerhalb von 21 Tagen) entwickelten sich signifikant seltener in der Lopinavir/Ritonavir-Gruppe als in den Kontrollgruppen ( $p<0,001)$. Außerdem zeigte die Studie in der Lopinavir/Ritonavir-Gruppe eine statistisch signifikante Reduktion des Einsatzes von Steroiden aufgrund akuter Verschlechterung der Lungenfunktion ( $p<0,001$ ) und eine signifikante Reduktion nosokomialer Infektionen im Vergleich zu den Kontrollgruppen ( $p<0,043$ ).

Mehrere Untersuchungen zeigten, dass der Verzicht auf eine Therapie mit Lopinavir/Ritonavir, fortgeschrittenes Alter (> 60 Jahre) sowie ein positiver Hepatitis B-Trägerstatus voneinander unabhängige Risikofaktoren für die Entwicklung eines schweren Krankheitsverlaufes waren.

Diese schweren Verläufe gingen einher mit der Entwicklung eines ARDS (Acute Respiratory Distress Syndrom) oder führten zum Tod [36]. Zusammenfassend zeigen die vorliegenden Studien, dass Lopinavir/Ritonavir ein effektives Kombinationstherapeutikum für die Therapie von SARS zu sein scheint.

Auch andere Proteaseinhibitoren wurden in vitro auf ihre antivirale Aktivität gegen das SARS-CoV geprüft. So testeten Yamamoto u. Mitarb. [38] eine Reihe von in der antiviralen Therapie bereits etablierten Substanzen und fanden heraus, dass Nelfinavir ein starker Replikationshemmer des SARS-CoV ist. Nelfinavir hemmte den durch Coronaviren induzierten zytopathischen Effekt und verringerte die Expression viraler Antigene in mit Nelfinavir therapierten Zellen. Auch Bernard u. Mitarb. [39] entdeckten zwei Proteaseinhibitoren (Calpain inhibitor VI/ III), die gegen das SARS-Coronavirus in vitro Wirksamkeit zeigten.

Proteaseinhibitoren scheinen gegen das SARS-CoV, zumindest experimentell, Wirksamkeit zu besitzen, und man kann davon ausgehen, dass es in diesem Gebiet noch weitergehende Studien geben wird.

\section{Virostatika - Virale Bindungsinhibitoren}

Die membranassoziierte Carboxypeptidase ACE2 (Angiotensin Converting Enzyme 2) ist ein zellulärer Rezeptor des SARS-Coronavirus. Er interagiert mit der S1-Domäne des SARS-CoV Spike Proteins [41]. Peptide oder andere Verbindungen, die an ACE2 binden, könnten daher möglicherweise in der Prävention und Therapie von SARS eingesetzt werden [42,43]. Eine lösliche Form des Rezeptors, Rezeptor-Antikörper oder die rezeptorbin- 
dende Domäne des Spike-Proteins könnten weitere Ansätze einer Therapie sein.

Sui u. Mitarb. [44] identifizierten in einer Bibliothek für nichtimmune humane Antikörper einen humanen monoklonalen antiS1-Antikörper, 80R, der durch Blockade der Virusbindung an den ACE2-Rezeptor der Wirtszelle eine Infektion mit dem SARS$\mathrm{CoV}$ verhinderte. Die Untersuchungen zeigten, dass der Antikörper mit dem löslichen ACE2 um die Bindung der S1-Domäne konkurrierte. Der Antikörper zeigte hierbei eine hohe Affinität zur S1-Domäne des SARS-CoV Spike-Proteins.

\section{Virostatika - Fusionsinhibitoren}

In vitro-Testergebnisse sowie theoretische Überlegungen weisen darauf hin, dass Fusionsinhibitoren in der Therapie von SARS effektiv sein könnten [44,45].

Peptide, die mit den HR („heptad repeat regions“) 1 und 2 des HIV-1 gp41 interagieren, sind die Grundlage der in der HIV-Therapie eingesetzten Fusionsinhibitoren. Gp41 ist ein transmembranes Virusprotein, das bei der Fusion des HIV mit der Wirtszelle eine elementare Rolle spielt. Aufgrund zahlreicher Ähnlichkeiten zwischen den „heptad repeat regions“ des gp41 und „heptad repeat regions“ des SARS-CoV Spike-Proteins ging man davon aus, dass diese Viren einen gemeinsamen Mechanismus der Fusion mit der Wirtszelle besitzen [44,45]. Liu u. Mitarb. [44] testeten 2 Reihen von Peptiden, die mit den „heptad repeat regions“ des Spike-Proteins interagierten, auf ihre hemmende Wirkung gegen das SARS-Coronavirus. Sie fanden heraus, dass das Peptid CP1 in vitro eine Infektion mit dem SARS-CoV verhindert. Man geht davon aus, dass CP1 an das HR1 des Spike-Proteins bindet und so die für die Fusion mit der Zielzelle benötigten Konformationsänderungen des Proteins verhindert. Erste in vivo Studien stehen noch aus.

\section{Virostatika - RNA-Interferenz}

Die Technik der RNA-Interferenz zur Inaktivierung von Genen wurde bereits in der Therapie von HIV, Hepatitis B und Hepatitis C experimentell angewendet [47 - 49]. Sie beruht auf der Einführung kurzer doppelsträngiger RNA-Moleküle (small interfering RNAs - siRNA) in eine Zelle oder einen Organismus. Diese RNAMoleküle entsprechen in ihrer Nukleotidsequenz der Sequenz des zu inaktivierenden Gens. Es kommt zur Hybridisierung der RNA-Moleküle mit der mRNA des Zielgens und in der Folge zum Abbau der mRNA. Um die Möglichkeit der Anwendung einer Gen-Inaktivierung in der SARS-Therapie zu prüfen, wurden spezifische kurze RNA-Moleküle synthetisiert, die dem SpikeGen des SARS-CoV entsprechen. Diese RNA-Moleküle hemmten effektiv und spezifisch die Expression des Spike-Proteins in SARS-CoV infizierten Zellen [50]. Eine weitere Studie untersuchte in vitro die Wirksamkeit von sechs kleinen RNA-Molekülen, die verschiedenen Abschnitten der Replikase 1A-Region des SARS-CoV entsprachen. Drei der RNA-Moleküle hemmten deutlich die zytopathischen Effekte, die durch eine Infektion und durch die Replikation des Virus hervorgerufen werden [51].

\section{Glycyrrhizin - Traditionelle Chinesische Medizin}

Glycyrrhizin ist ein Bestandteil der Lakritzwurzel. Es hemmt die Replikation des HIV in vitro [52] und wurde, teilweise mit Erfolg, bereits in der Therapie von Hepatitis C [53] und Hepatitis B [54] eingesetzt. In vitro-Studien haben gezeigt, dass Glycyrrhizin auch die Replikation des SARS Coronavirus hemmt [33]. Der Mechanismus der durch Glycyrrhizin induzierten Hemmung der Virusreplikation ist bisher jedoch nicht bekannt. Eine wichtige
Rolle könnte ein antiviraler Effekt des Stickstoffmonoxid (NO) spielen. Glycyrrhizin erhöht in diesem Zusammenhang die Expression der NO-Synthetase und die Produktion von NO in Mausmakrophagen [55]. Auch Ergebnisse von Cinatl u. Mitarb. [33] zeigen, dass Glycyrrhizin in Affennierenzellen (die zur Kultivierung des SARS-Coronavirus verwendet wurden) die Expression der NO-Synthetase induzierte.

\section{Stickstoffmonoxid (NO)}

Cinatl u. Mitarb. [33] zeigten, dass die Replikation des SARS-CoV gehemmt wird, wenn „DETA NONOate“ - ein NO-Donor - zum Kulturmedium hinzugefügt wird. Dieses Ergebnis wurde durch Keyaerts u. Mitarb. bestätigt [56], die als NO-Donor S-nitro$\mathrm{N}$-acetyl-Penicillamin verwendeten. Außerdem untersuchten Keyaerts u. Mitarb. an SARS-Patienten die Wirksamkeit einer Inhalationstherapie mit NO-Gas. Die Erkrankten zeigten sofort nach Verabreichung eine Verbesserung der Oxygenierung. Die Wirkung hielt außerdem über die Beendigung der Inhalation hinaus an. Der zugrunde liegende Effekt beruht wahrscheinlich auf einer NO-bedingten Vasodilatation der pulmonalen Kapillaren im Bereich ventilierter Areale. Dieser Effekt wird generell bei ARDS beobachtet, und die Wirkungen NOs haben deswegen wahrscheinlich weniger mit dem direkten antiviralen Effekt zu tun, der von Cinatl u. Mitarb. in vitro nachgewiesen wurde.

\section{Niclosamid}

Wu u. Mitarb. testeten in vitro eine Reihe von Substanzen, die bisher nicht als Virustatika auf dem Markt registriert waren, auf ihre antivirale Aktivität gegen das SARS-Coronavirus. Sie fanden heraus, dass Niclosamid - eigentlich ein Anthelmintikum - die Replikation des SARS-CoV hemmt. Der virustatische Wirkmechanismus ist unbekannt. Die Studie zeigte aber, dass Niclosamid weder die Adsorption oder das Eindringen in die Wirtszelle noch die Aktivität der Protease beeinflusste.

\section{Immunmodulatoren - Steroide}

Während des Ausbruchs von SARS Ende 2002 bis Anfang 2003 gehörte die systemische Gabe von Steroiden zu den Hauptmaßnahmen in der SARS-Therapie [58]. Die Gründe hierfür lagen u.a. in der Beobachtung, dass trotz eines Absinkens der Viruslast und Anstiegs der SARS-CoV spezifischen IgG-Ak in der 3. Krankheitswoche bei einigen Patienten paradoxerweise eine Verschlechterung des Krankheitszustandes eintrat [30]. Pathologische Untersuchungen zeigten Bronchitis-obliterans-Pneumonien und akutes Lungenversagen. Diese Befunde führten zu der Hypothese, dass es während einer SARS-CoV-Infektion zu einer immunologischen Hyperaktivität des Wirtes und so zu einer zytokinvermittelten Lungenschädigung kommt. Diese Hyperaktivität des Immunsystems könnte durch eine Steroidtherapie reduziert werden [59].

In den meisten Fällen wurden Steroide ergänzend zu einer Ribavirintherapie verabreicht. Verschlechterte sich die Lungenfunktion des Patienten, wurden außerdem hoch dosierte Steroide gegeben. Studien, die die Effektivität einer Therapie mit Steroiden untersuchten, beinhalteten fast immer die gleichzeitige Gabe von mehreren Therapeutika. Außerdem enthielt keine der Studien eine Kontrollgruppe. Inwieweit Steroide also tatsächlich in der Therapie von SARS wirksam sind, kann noch nicht eindeutig festgestellt werden.

In einigen Studien schienen Therapieregime, die Steroide enthielten, mit Verbesserungen der Thorax-Röntgen Befunde, Fie- 
berabfall und einer schnelleren Verbesserung der Oxygenierung im Zusammenhang zu stehen [31,60,61].

In einer Studie von Hsu u. Mitarb. [61] zeigte die zusätzliche Gabe von Steroiden jedoch keine klinischen Verbesserungen. Allerdings wurde in dieser Studie eine niedrigere Steroiddosis verabreicht als in den Studien, die eine klinische Besserung aufzeigten.

Ho u. Mitarb. [62] verglichen in einer retrospektiven Studie die klinischen und radiologischen Daten von möglichen SARS-Patienten, die mit Ribavirin behandelt wurden. Siebzehn dieser Patienten bekamen zusätzlich initial eine Stoßtherapie mit hoch dosierten Steroiden, 55 der Patienten bekamen initial niedrig dosierte Steroide. Hoch dosierte Steroide wurden außerdem an Patienten mit einer akuten Verschlechterung der Lungenfunktion verabreicht. Die kumulative Steroiddosis, die Zahl der Intensivstationsaufnahmen, der Bedarf an künstlicher Beatmung sowie die Mortalitätsrate waren nach 21 Tagen in beiden Patientengruppen gleich. Allerdings benötigten die Patienten, denen initial eine Hochdosis-Stoßtherapie verabreicht wurde, weniger Sauerstoff und zeigten früher eine Verbesserung der radiologischen Befunde. Außerdem hatten diese Patienten deutlich weniger Bedarf an einer Notfallmedikation mit Steroiden. Das Ergebnis dieser Studie zeigt, dass durch eine frühe Gabe von Steroiden in Form einer Hochdosis-Stoßtherapie möglicherweise eine deutliche Verbesserung im Krankheitsverlauf von SARS-Patienten erreicht werden kann.

Allerdings werden Studien mit eindeutigeren Ergebnissen und einem Einbezug von Kontrollgruppen benötigt. Außerdem sollte zwischen dem Nutzen einer Steroidtherapie und den damit verbundenen Risiken, wie der Entwicklung avaskulärer Nekrosen, einer sekundären Sepsis oder einer disseminierten Aspergillose abgewogen werden. Einige dieser verheerenden Nebenwirkungen wurden bei SARS-Patienten bereits beobachtet $[63,64]$. Hong u. Mitarb. [65] untersuchten zwischen März und Mai 2003 in Beijing 67 SARS-Patienten, die mit Ribavirin und Steroiden behandelt wurden und an starken Gelenkschmerzen litten, die wahrscheinlich durch avaskuläre Nekrosen hervorgerufen wurden. Bei 28 Patienten (42\%) zeigten die Röntgen- und MRTAufnahmen eine Bestätigung des Verdachtes. Die Diagnose der avaskulären Nekrose wurde im Durchschnitt 4 Monate nach Beginn der Steroideinnahme gestellt.

\section{Immunmodulatoren - Interferone}

In vitro-Studien haben gezeigt, dass Typ 1-Interferone die Replikation des SARS-Coronavirus hemmen [32,66-68]. Da diese Studienergebnisse bereits während der SARS-Epidemie vorlagen, wurden Interferone im weiteren Verlauf der Epidemie klinisch eingesetzt.

Loutfy u. Mitarb. [69] dokumentierten ihre klinischen Erfahrungen, die sie mit der Therapie mit Interferon alfacon 1 (einem rekombinanten synthetischen Typ 1-Interferon) bei 22 SARS-Patienten in einer Studie in Toronto machten. 13 Patienten, die nur mit Steroiden behandelt wurden, wurden mit 9 Patienten, die Steroide und Interferon alfacon 1 erhielten, verglichen. Die Patientengruppe, die zusätzlich mit Interferon alfacon 1 behandelt wurde, zeigte eine signifikante Verbesserung der Sauerstoffsättigung $(p=0,02)$ und bessere radiologische Befunde. Außerdem zeigte diese Patientengruppe deutlich geringere Anstiege der Kreatinkinase und eine schnellere Normalisierung der Lactatdehydrogenasekonzentration [69]. Jedoch erhielt die Interferon alfacon-Gruppe auch höhere Dosen an Steroiden, so dass nicht eindeutig festgestellt werden kann, ob die besseren
Ergebnisse dieser Gruppe tatsächlich auf die Wirkung des Interferon alfacon 1 zurückgeführt werden können.

Haagmans u. Mitarb. [70] untersuchten die prophylaktische Anwendung von Interferonen in einem Affenmodell. 3 Tage vor der Einimpfung des SARS-CoV wurde einem Teil der Affen pegyliertes Interferon alfa verabreicht. Diese Affen zeigten im Gegensatz zu den nicht behandelten Affen eine deutliche Reduktion der Replikation und Freisetzung des Virus, eine verminderte Expression viraler Antigene in Typ 1-Pneumozyten und eine geringere Schädigung der Lunge. Postexpositionelle Behandlungen mit pegyliertem Interferon alfa zeigten weniger deutliche Ergebnisse.

\section{Diskussion}

Die zentrale Frage der heutigen Forschung ist die nach Strategien für den Fall einer neuen SARS-Epidemie. In der Zeit des Ausbruchs von SARS wurden zahlreiche Therapeutika gegen SARS eingesetzt, jedoch erfolgte dies in keinem Fall klinisch kontrolliert.

Basierend auf den bis heute vorliegenden Daten über Substanzen mit guten klinischen Ergebnissen scheinen - von den sofort verfügbaren Therapeutika - Typ 1-Interferone, Steroide und die Kombinationstherapie aus Lopinavir/Ritonavir die größte Wirkung gegen eine SARS Coronavirus Infektion zu zeigen.

Idealerweise sollten alle potenziell wirksamen Substanzen in kontrollierten klinischen Studien evaluiert werden. Jedoch kam es bereits während der SARS-Epidemie zu zahlreichen Schwierigkeiten in der Gestaltung und Ausführung klinischer Studien, die u.a. von Muller u. Mitarb. [71] zusammengefasst wurden. Im Falle eines plötzlichen Ausbruchs einer neuen Erkrankung durch einen unbekannten Erreger stehen viele Daten und Informationen, die zur Durchführung klinischer Studien benötigt werden, nicht zur Verfügung. Hierzu gehören u.a. spezifische mikrobiologische Tests zur Identifizierung einer geeigneten Studienpopulation und Richtlinien, die Dosis, Frequenz und Dauer einer therapeutischen Intervention festlegen. Ein weiteres Problem besteht darin, eine ausreichende Zahl an Patienten zu rekrutieren, bevor eine Epidemie endet bzw. Patienten frühzeitig aus der Studie herausfallen. Die Möglichkeit, eine Studie schnell planen und durchführen zu können, ist jedoch abhängig von der Zeit, die benötigt wird, bis eine Bewilligung durch die Ethikkommission erfolgt ist und ausreichende Fördermittel zur Verfügung stehen.

Die Gründung einer international zusammenarbeitenden Arbeitsgruppe zur Planung und Durchführung klinischer Studien mit Zugriff auf einen Hilfsfonds und der Basis international akzeptierter ethischer Grundsätze könnte eine schnelle Ausführung klinischer Studien im Fall einer neuen Epidemie erleichtern.

\section{Fazit für die pneumologische Praxis}

SARS ist eine neu entdeckte Viruserkrankung, die sich im Jahre 2002/2003 von Südchina ausgehend weltweit ausbreitete und zu über 700 Todesfällen führte. Innerhalb kürzester Zeit konnte ein neues Coronavirus - das SARS-Coronavirus - als auslösender Erreger identifiziert werden. Eine spezielle Katzenart aus Südchina wurde als mögliches tierisches Reservoir des Virus bestimmt. Eine Infektion mit dem SARS-Coronavirus führt zu relativ unspezifischen Symptomen wie Myalgien, Fieber, Husten und 
Tab. 2 Empfehlungen des Robert Koch-Instituts zum Umgang mit Probenmaterial von Patienten mit Verdacht auf schweres akutes Atemwegssyndrom (SARS)

- Bei Blutproben grundsätzliches Vorgehen zunächst wie bei HBV-,
HCV-, HIV-Infektion.
- Alle Maßnahmen, die mit Eröffnung von Probengefäßen verbunden
sind, sollten unter einer Laminar-Air-Flow-Sicherheitswerkbank Klas-
se 2 durchgeführt werden. Dabei sind Schutzkittel, Handschuhe so-
wie Mund- und Nasenschutz (FFP2/FFP3) und Schutzbrille zu tragen.
- Nach allen Arbeiten abschließend Händedesinfektion.
- Dekontamination der Analysegeräte, falls erforderlich, nach Vor-
gaben des Herstellers. Bei fehlenden Angaben am ehesten unter Ver-
wendung von $90 \%$ Äthanol.
- Im Übrigen Beachtung der TRBA 100 (Schutzmaßnahmen für gezielte
und nicht gezielte Tätigkeiten mit biologischen Arbeitsstoffen).
- Abfallentsorgung gemäß der Richtlinie über die ordnungsgemäße
Entsorgung von Abfällen aus Einrichtungen des Gesundheitswesens
(LAGA).

Dyspnoe. Die am meisten gefürchtete Komplikation ist eine schwere Lungenentzündung und die Entwicklung eines akuten Lungenversagens.

Im Verlauf der Epidemie wurden verschiedene Substanzen gegen SARS eingesetzt, jedoch ohne Einbezug in kontrollierte klinische Studien. Daher ist noch keine zuverlässige Therapie gegen das SARS-Coronavirus gefunden. Seit dem Ausbruch wurden zahlreiche Studien zur Evaluierung neuer Therapiemöglichkeiten durchgeführt. Ergebnisse von In-vitro-Tests und Tiermodellen zeigten bei einigen Substanzen eine gute Wirksamkeit gegen das SARS-Coronavirus.

Ganz praktisch sollten im Fall eines erneuten Ausbruchs von SARS viel versprechenden Substanzen und Impfstoffe in kontrollierten klinischen Studien evaluiert werden. Dies könnte durch die Gründung einer internationalen Arbeitsgruppe für klinische Studien erleichtert werden. Diese sollte spezielle Privilegien besitzen, um auch während einer schnell voranschreitenden Epidemie die Planung und Durchführung klinischer Studien zu ermöglichen. Im Falle von Verdachtsmomenten sollten die jeweils aktuellen Informationen des Robert Koch-Instituts abgerufen werden unter http://www.rki.de und die Empfehlungen zum Umgang mit Probenmaterial von Patienten mit Verdacht auf SARS strikt beachtet werden ( Tab. 2). Hilfreich ist auch eine Konsultation des US-amerikanischen Centers for Disease Control and Prevention (http://www.cdc.gov).

\section{Institutsangaben}

Abteilung Pneumologie, Zentrum Innere Medizin, Medizinische Hochschule Hannover (Direktor: Univ.-Prof. Dr. T. Welte)

2 Zentrum für Pneumologie und Thoraxchirurgie Heckeshorn, Klinik für Pneumologie, HELIOS Emil-von-Behring, Berlin (Chefarzt: PD Dr. T. T. Bauer) Forschungszentrum Borstel, Medizinische Klinik, Borstel (Direktor: Univ.Prof. Dr. P. Zabel)

${ }^{4}$ Medizinische Klinik III, Universitätsklinikum Schleswig-Holstein, Campus Lübeck, Universität zu Lübeck (Direktor: Univ.-Prof. Dr. P. Zabel)

\section{Literatur}

1 Groneberg DA, Poutanen SM, Low DE et al. Treatment and vaccines for severe acute respiratory syndrome (SARS). Lancet Infect Dis 2005; 5: $147-155$

2 Poon LL, Guan Y, Nicholls YM et al. The aetiology, origins and diagnosis of severe acute respiratory syndrome. Lancet Infect dis 2004; 4: 663 671

3 Reilley B, Herp M van, Sermand D et al. SARS and Carlo Urbani. N Engl J Med 2003; 348: 1951 - 1952

4 World Health Organization (WHO). Summary of propable SARS cases with onset of illness from 1. November 2002 to 31. July 2003. http// www.who.int/csr/sars/country/table2004_04_21/en, 2003 (accessed feb 3, 2005)

5 World Health Organization Multicentre Collaborative Network for Severe Acute Respiratory Syndrome Diagnosis. A multicentre collaboration to investigate the cause of severe acute respiratory syndrome. Lancet 2003; 361: 1730-1733

6 Peiris JS, Lai ST, Poon LL. Coronavirus as a possible cause of severe acute respiratory syndrome. Lancet 2003; 361: 1319-1325

7 Drosten C, Gunter S, Preiser W. Identification of a novel coronavirus in patients with severe acute respiratory syndrome. N Engl J Med 2003; 348: 1967 - 1976

8 Ksiazek TG, Erdmann D, Goldsmith CS. A novel coronavirus associated with severe acute respiratory syndrome. N Engl J Med 2003; 348: 1953 - 1966

9 Zhang QF, Cui JM, Huang XJ. Morpholigy and morphogenesis of severe acute respiratory syndrome (SARS)-associated virus. Chin J Biochem Biophys 2003; 35: 587 - 591

10 Cavanagh D. Nidovirales: a new order comprising Coronaviridae and Arteriviridae. Arch Virol 1997; 142: 629-633

11 Sidell S, Wege H, ter Meulen $V$. The structure and replication of coronaviruses. Curr Top Microbiol Immunol 1982; 99: 131 - 163

12 Wege H, Sidell S, ter Meulen V. The biology and pathogenesis of Coronaviruses. Curr Top Microbiol Immunol 1982; 99: 165-200

13 Guan Y, Zheng BJ, He YQ. Isolation and characterization of viruses related to the SARS coronavirus from animals in southern China. Science 2003; 302: $276-278$

14 Bonn D. Wild animals could be source of SARS. Lancet Infect Dis 2003 3: 395

15 Chan PKS, Tang JW. SARS: clinical presentation, transmission, pathogenesis and treatment options. Clinical Science 2006; 110: $193-204$

16 Dietel M, Suttorp N, Zeitz M. Harrisons Innere Medizin, 16. Aufl. ABWWissenschaftsverlag, 2005

17 Marra MA, Jones SJ, Astell CR. The genome sequence of the SARS-associated coronavirus. Science 2003; 300: 1399-1404

18 Groneberg DA, Hilgenfeld $R$, Zabel P. Molecular mechanisms of severe acute respiratory syndrome (SARS). Respir Res 2005; 6: 8

19 Lai MM, Cavanagh D. The molecular biology of coronaviruses. Adv Virus Res 1997; 48: 1 - 100

20 Wentworth DE, Gillim-Ross L, Espina $N$ et al. Mice susceptible to SARS coronavirus. Emerge Infect Dis 2004; 10: 1293-1296

21 Martina BE, Haagmans BL, Kuikan T. Virology: SARS virus infection of cats and ferrets. Nature 2003; 425: 915

22 Tsui PT, Kwok ML, Yuen H et al. Sevre acute respiratory syndrome: clinical outcome and prognostic correlates. Emerg Infect Dis 2003; 9: 1064- 1069

23 Donnelly CA, Ghani AC, Leung GM. Epidemiological determinants of spread of causal agent of severe acute respiratory syndrome in Hong Kong. Lancet 2003; 361: 1761 - 1766

24 Bitnun A, Allen $U$, Heurter $H$. Children hospitalized with severe acute respiratory syndrome - related illness in Toronto. Pediatrics 2003; 112: e261

25 Zhaori G. Antiviral treatment of SARS: Can we draw any conclusions? CMAJ 2003; 169: 1165-1166

26 Hof H, Müller RL, Dörris R. Mikrobiologie-Duale Reihe. 1. Aufl. Stuttgart/D, 2000

27 Crotty S, Maag D, Arnold JJ. The broad-Spectrum antiviral ribonucleoside ribavirin is an RNA virus mutagen. Nat Med 2000; 6: 1375 - 1379

28 Zhang KW, Ho PL, Ooi GC. A cluster of cases of severe acute respiratory syndrome in Hong Kong. N Engl J Med 2003; 348: 1977-1985

29 Lee $\mathrm{N}$, Hui $\mathrm{D}, \mathrm{Wu} \mathrm{A}$. A major outbreak of severe acute respiratory syndrome in Hong Kong. N Engl J Med 2003; 348: 1986-1994

30 Peiris JS, Chu CM, Cheng VC. Clinical progression and viral load in a community outbreak of coronavirus-associated SARS pneumonia: a prospective study. Lancet 2003; 361: 1767-1772 
31 Zhao Z, Zhang F, Xu M. Description and clinical treatment of an early outbreak of severe acute respiratory syndrome (SARS) in Guangzhou, PR China. J Med Microbiol 2003; 52: 715 - 720

32 Stroher $U$, DiCaro A, Li Y. Severe acute respiratory syndrome-related coronavirus is inhibited by interferon-alfa. J Infect Dis 2004; 189: $1164-1176$

33 Cinatl J, Morgenstern B, Bauer G et al. Glycyrrhizin, an active component of liquorrice roots, and replication of SARS-associated coronavirus. Lancet 2003; 361: 2045 - 2046

34 Mazzulli T, Farcas GA, Poutanan SM. Sevre acute respiratory syndrome-associated corornavirus in lung tissue. Emerge Infect Dis 2004; 10: $20-24$

35 Knowles SR, Phillips EJ, Dresser L et al. Common adverse events associated with the use of Ribavirin for severe acute respiratory syndrome in Canada. Clin Infect Dis 2003; 37: 1139-1142

36 Zhu CM, Cheng VC, Hung IF. Role of Lopinavir/Ritonavir in the treatment of SARS: Initial virological and clinical findings. Thorax 20004 ; 59: $252-256$

37 Chan KS, Lai ST, Chu CM. M. Treatment of SARS with Lopinavir/Ritonavir: A multicentre retrospective matched cohort study. Hong Kong Med J 2003; 9: 399-406

38 Yamamoto $N$, Yang $R$, Joshinaka $Y$. HIV protease inhibitor nelfinavir inhibits replication of SARS-associated coronavirus. Biochem Biophys Res Commun 2004; 318: 719- 725

39 Barnard DL, Hubbart VD, Burten J. Inhibition of SARS-associated coronavirus (SARSCOV) by calpain inhibitors in beta-D-N4-hydroxycytidine. Antivir Chem Chemother 2004; 15: 15 - 22

$40 \mathrm{Li} \mathrm{W}$, Moore MJ, Vasilieva N. Angiotensin-converting enzyme is a functional reseptor for the SARS coronavirus. Nature 2003; 426: $450-454$

41 Huang L, Sexton DJ, Skogerson K. novel peptide inhibitors of angiotensin-converting enzyme2. J Biol Chem 2003; 278: 15532-15540

42 Dales NA, Gould AE, Brown JA. Substrate based design of the first class of angiotensin-converting enzyme-related carboxypeptidase (ACE2) inhibitors. J Am Chem Soc 2002; 124: 11852 - 11853

43 Sui J, Li W, Murakami A. Potent neutralisation of SARS coronavirus by a human $\mathrm{mAb}$ to $\mathrm{S} 1$ protein that blocks reseptor association. Prog Natl Acad Sci USA 2004; 101: 2536-2541

44 Liu S, Xiao G, Chen $Y$. Interaction between heptad repeat 1 and 2 regions in spike protein of SARS-associated coronavirus: implications for virus fusogenic mechanism and identification of fusion inhibitors. Lancet 2004; 363: 938 - 947

45 Kliger Y, Levanon EY. Cloaked similarity between HIV-1 and SARS-CoV suggests an anti-SARS strategy. BMC Microbiol 2003; 3: 20

46 Zhang $R$, Guo Z, Lu J. Inhibiting SARS-associated coronavirus by small interfering RNA. Chin Med J 2003; 116: 1262 - 1264

47 Jacque JM, Triques K, Stevenson M. Modulation of HIV-1 replication by RNA interference. Nature 2002; 418: 435 - 438

48 Kabadia SB, Brideau-Anderson A, Chisari FV. Interference of hepatitis C virus RNA replication by short interfering RNAs. Proc Natl Acad Sci USA 2003; 100: 2014-2018

49 Hamasaki $K$, Nakao $K$, Mazumoto $K$ et al. Short interfering RNA-directed inhibition of hepatitis $B$ virus replication. FEBS Lett 2003; 543: $51-54$

50 Zhang J, Li T, Fu L. Silencing SARS-CoV spike protein expression in cultured cells by RNA interference. FEBS Lett 2004; 560: 141 - 146
51 He ML, Zheng B, Peng Y. Inhibition of SARS-associated coronavirus infection and replication by RNA interference. JAMA 2003; 290: 2665 2666

52 Sasaki H, Takai M, Kobajashi M et al. Effect of Glycyrrhizin, an activ component of liquorrice roots, on HIV replication in cultures of peripheral blood mononuclear cells from HIV-seropositive patients. Pathobiology 2002; 70: 229-236

53 Kumada $\mathrm{H}$. Lon-term treatment of chronic hepatitis C with Glycyrrhizin [stronger neo-minophagen C (SNMC)] for preventing liver cirrhosis and hepatocellular carcinoma. Oncology 2002; 62 (suppl 1): $94-$ 100

54 Matsuo K, Takenaka K, Chimomura H. Lamivudine and Glycyrrhizin for treatment of chemotherapy-induced hepatitis B virus (HBV) hepatitis in a chronic HBV carrier with non-hodgkin lymphoma. Leuk Lymphoma 2001; 41: 191 - 195

55 Kim Jy, Choi CY, Lee KJ. Induction of inducible nitric oxide synthase and proinflammatory cyrokines expression by o, p'-DDT in macrophages. Toxicol Lett 2004; 147: 261 - 269

56 Keyaerts E, Vijgen L, Maes $P$ et al. Inhibition of SARS-CoV infection in vitro by S-nitroso-N-acetylpenicillamine, a nitric oxid donor compound. 11th International Congress on Infectious Diseases Cancun, Mexico, March 4-7, 2004

$57 \mathrm{Wu} C \mathrm{CJ}$, Jan JT, Chen CM. Inhibition of SARS coronavirus replicaton by niclosamide. Antimicrob Agents Chemother 2004; 48: 2693 - 2696

58 Gomersall TD, Kargel MJ, Lapinski SE. Pro/Con clinical debate: steroids are a key component in the teratment of SARS. Crit Care 2004; 8: 105 107

59 Nicholls JM, Poon LL, Lee KC. Lung pathology of fatal SARS. Lancet 2003; 361: 1773 - 1778

60 Booth TM, Matukas LM, Tomlinson GA. Clinical features and short-term outcomes of 144 patients with SARS in the greater Toronto area. JAMA 2003; 289: 2801 - 2809

61 Hsu LY, Lee CG, Green JA. SARS in Singapore: clinical features of index patient and initial contacts. Emerg Infect Dis 2003; 9: 713-717

62 Ho JC, Ooi GC, Mok TY. High-dose pulse versus nonpulse corticosteroid regiments in SARS. Am J Respir Crit Cer Med 2003; 168: 1449 - 1456

63 Wang H, Ding Y, Li X et al. Fatal aspergillosis in a patient with SARS who was treated with corticosteroids. N Engl J Med 2003; 349: 507 508

64 Chan KS, Zheng JP, Mok YW. SARS: prognosis, outcome and sequelae. Respirology 2003; 8 (suppl): S36-40

65 Hong N, Du XK. Avascular necrosis of bone in SARS. Clin Radiol 2004 59: $602-608$

66 Cinatl JR, Michaelis M, Scholz $M$ et al. Role of interference in the treatment of SARS. Expert Opin Biol Ther 2004; 4: 827-836

67 Tan EL, Ooi EE, Lin CY. Inhibition of SARS coronavirus infection in vitro with clinically approved antiviral drugs. Emerg Infect Dis 2004; 10: $581-586$

68 Hensley LE, Fritz LE, Jahraling PB et al. Interferon- $\beta$ 1a and SARS coronavirus replication. Emerg Infect Dis 2004; 10: 317 - 319

69 Loutfy MR, Blatt LM, Siminovich KA. Intereferon alfacon-1 plus corticosteroids in SARS: a preliminary study. JAMA 2003; 290: $3222-3228$

70 Haagmanns BL, Kuiken T, Martina BE. Pegylated interferon-alpha protects type 1 pneumocytes against SARS coronavirus infection in macaques. Nat Med 2004; 10: 290-293

71 Muller MP, McGeer A, Straus SE et al. Clinical trials and novel pathogens: lessons learned from SARS. Emerg Infect Dis 2004; 10: 389 - 394 